\title{
Automatic Detection of Erythrocytes in Fishes using Clustering Segmentation and Supervised Learning
}

\author{
Kleyton Sartori Leite*, Felipe Gomes da Silva*, Bruno do Amaral Crispim ${ }^{\dagger}$, \\ Felipe Merey ${ }^{\dagger}$, Alexeia Barufatti Grisolia ${ }^{\dagger}$ and Willian Paraguassu Amorim* \\ *Faculdade de Ciências e Tecnologia - UFGD, Dourados, MS \\ Email: kleyton.sartori.leite@gmail.com, felipe.gomess.1993@gmail.com, willianamorim@ufgd.edu.br \\ ${ }^{\dagger}$ Faculdade de Ciências Biológicas e Ambientais - UFGD, Dourados, MS \\ Email: felipemendesmerey@hotmail.com, brunocrispim.bio@gmail.com, alexeiagrisolia@ufgd.edu.br
}

\begin{abstract}
Resumo-The growth of urban areas and the population has favored the increase in pollution and consequently the contamination of river waters. This clue has aroused interest in several aspects, mainly related to the fate and possible effects that these contaminants can cause to human health. The analysis of erythrocytes in fish is an efficient mechanism to identify the presence of genetic alterations that may be being caused by emergent contaminants. This article presents a new proposal for automatic identification of erythrocytes in fish using SLIC segmentation approach and connected components, adjusted using supervised learning, and presenting the performance evaluation in different aspects of the image.
\end{abstract}

\section{INTRODUÇÃO}

O ser-humano tem alterado constantemente o meio ambiente, seja no meio rural com a atividade agropecuária, modificando os processos dos sistemas naturais [1] ou nas cidades, com a presença de esgoto doméstico, lixo e carga urbana difusa de poluição [2]. Um dos meios mais afetados é o aquático, onde contaminantes podem ser absorvidos facilmente pelo organismo, mesmo em baixas concentrações, permanecendo em cadeias tróficas por longos períodos. Essas mudanças retornam aos indivíduos efeitos colaterais preocupantes.

Dessa forma a aplicação de metodologias ou ferramentas que possam avaliar propriedades genotóxicas de corpos d'água causadas por contaminantes ambientais são prioritárias para gerar informações que auxiliaram na elaboração de planos de controle da qualidade da água. Com a utilização de bioindicadores de poluição ambiental é possível avaliar os efeitos causados por essas substâncias [10], pois estes são sensíveis à agentes poluidores e capazes de detectar misturas de contaminantes presentes no ambiente. Trabalhos recentes, mostram que análise de eritrócitos em testes utilizando peixes, animais que possuem eritrócitos nucleados [20], são eficientes por responderem aos componentes tóxicos de modo similar ao dos grandes vertebrados. Além disso, estes animais são encontrados abundantemente nos ecossistemas, possibilitando a identificação de contaminantes emergentes [21].

Atualmente, a aplicação desta metodologia para quantificar eritrócitos, é realizada manualmente, à exemplo do Laboratório de Ecotoxicologia e Genotoxicidade (LECOGEN). A Figura 1. apresenta o ambiente de avaliação e contagem.

Contudo, a identificação de eritrócitos manualmente por especialistas acarreta em inúmeras desvantagens, como: cansaço,

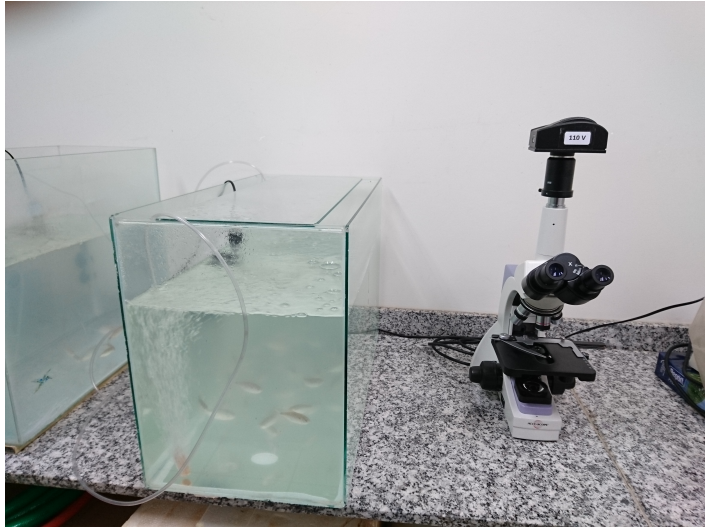

(a)

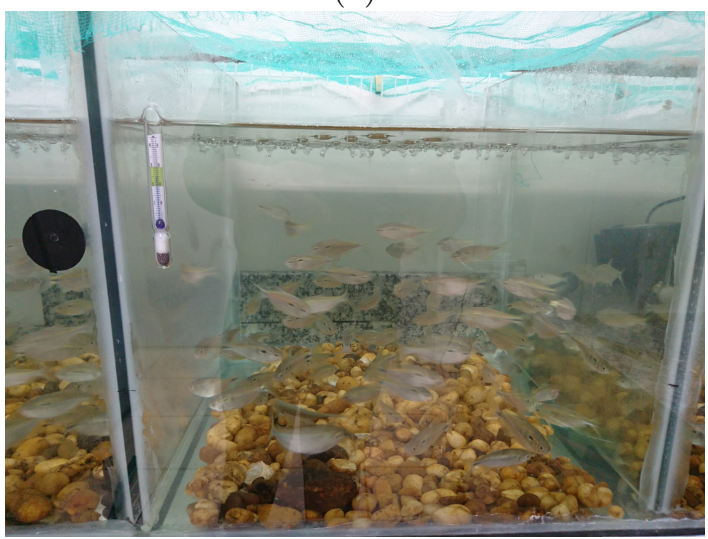

(b)

Figura 1. Equipamentos utilizados pelo Laboratório de Ecotoxicologia e Genotoxicidade: (a) áquarios com peixes em experimentação e microscópio para obtenção das imagens e (b) controle da água para manter estado original do ambiente do peixe.

devido à concentração necessária para a tarefa e a quantidade de amostras, que muitas vezes ultrapassam centenas de dados para análise; troca de especialistas, em alguns casos necessária para contagem para posterior validação dos resultados; limitação das análises laboratoriais que podem atrasar o término do processo e a liberação dos resultados, entre outros fatores que afetam os profissionais a provocar suscetíveis erros.

Durante os últimos anos várias técnicas foram criadas 
com o objetivo de auxiliar tarefas de detecção e contagem automáticas de objetos em imagens, com a garantia de resultados confiáveis. Como exemplo: Transformada de Hough, apresentada por Bewes e Mckenzie [3], aplicando a técnica na contagem de glóbulos brancos para determinar tipo de leucemia dos pacientes; Byun et al [4] apresentam a contagem das células de retinas usando Filtro Laplaciano de Gauss; e Souza et al. [5] utilizando Template Matching para a identificação de colônias bacterianas auxiliando o diagnóstico da infecção urinária.

Neste trabalho, propomos uma abordagem para a detecção e contagem automática em eritrócitos de peixes com o auxílio de aprendizagem de máquina. Basicamente, nossa proposta, recebe uma imagem de entrada, em seguida, superpixels são gerados pela técnica SLIC (simple linear iterative clustering) [6]. Esses superpixels, por sua vez, serão submetidos a uma classificação, que retornará dados para um grafo de componentes conectados [7] agrupando os superpixels com maior similaridade, para posterior identificação e contagem dos superpixels-eritrócitos.

O trabalho está organizado da seguinte forma. As Seções II e III apresentam o funcionamento das técnicas SLIC e a proposta para identificação de eritrócitos, respectivamente. A Seção IV, apresenta os experimentos realizados e os resultados obtidos. Por fim, a Seção V apresenta as conclusões obtidas a partir das implementações e experimentos realizados.

\section{SLIC SUPERPIXELS}

Superpixels é uma abordagem para segmentação de imagens em agrupamentos de pixels vizinhos com maior similaridade. Esse tipo de representação da imagem nos fornece uma melhor eficiência para os métodos de classificação, pois reduz a quantidade de primitivas a serem analisadas e permite a análise de características da imagem por regiões maiores.

Há várias técnicas para de segmentar imagens, tais como: segmentação baseada em grafos [8], segmentação por fluxo de borda [9], watershed [11], entre outras. Contudo, uma das mais citadas pela literatura é a SLIC (Segmentation Linear Iteractive Clustering) [6]. Basicamente, a SLIC divide a imagem em regiões com número aproximado de pixels, também chamados de superpixels. Na prática seu desempenho é melhor que outras técnicas já citadas, porque além de utilizar uma quantidade menor de memória, consegue realizar uma ótima aderência nas bordas, e mantém as fronteiras bem definidas entre os objetos da imagem.

A técnica SLIC, realiza um agrupamento local dos pixels, combinando o CIELAB, com a posição $(\mathrm{x}, \mathrm{y})$ dos pixels na imagem, gerando um espaço de cores de 5 dimensões, e usa uma adaptação do algoritmo Euclidiano para considerar o tamanho dos superpixels.

Seja $k$ a quantidade de superpixels desejada, o algoritmo (SLIC), inicia amostrando $k$ centros de grupos, regularmente espaçados, calculado por posições de gradiente em um intervalo de grade $S$. Cada pixel é associado ao grupo mais próximo (desde que a área seja sobreposta a esse pixel). Depois de todos os pixels estarem associados, é gerado um novo centro, usando CIELAB médio, baseado no novo grupo de pixels [6], Equação 3 , onde $D_{s}$ é a soma da distância $(L, A, B)$, pelo plano $(x, y)$, normalizado em $S$ e $m$ o coeficiente de proximidade espacial do agrupamento (quanto maior o $\mathrm{m}$, mais compacto é o cluster).

$$
\begin{gathered}
d_{(L, A, B)}=\sqrt{\left(L_{k}-L_{i}\right)^{2}+\left(A_{k}-A_{i}\right)^{2}+\left(B_{k}-B_{i}\right)^{2}} \\
d_{(x, y)}=\sqrt{\left(x_{k}-x_{i}\right)^{2}+\left(y_{k}-y_{i}\right)^{2}} \\
D_{s}=d_{L A B}+\left(\frac{m}{S}\right) * d_{X Y}
\end{gathered}
$$

Após esse processo, alguns rótulos podem estar perdidos (pixels que não foram adicionados a nenhum cluster), logo tais segmentos são rotulados novamente de acordo com os maiores grupos vizinhos [6]. A Figura 2 apresenta resultados de segmentação SLIC, usando diferentes quantidade de segmentos.

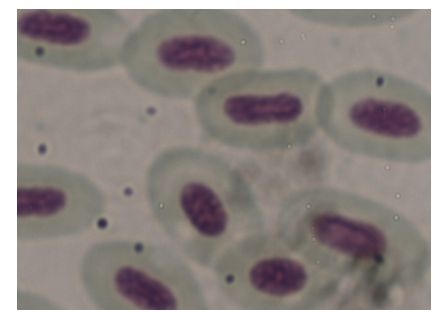

(a)

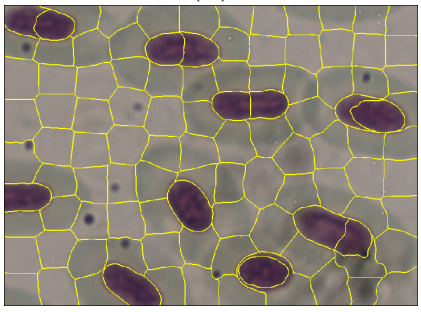

(c)

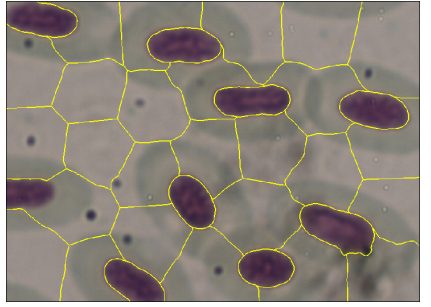

(b)

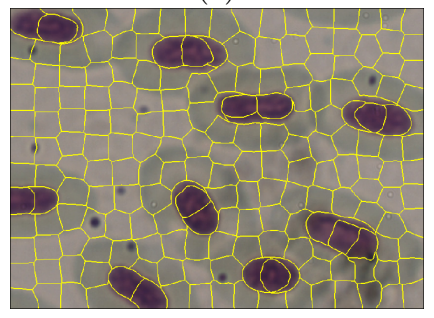

$(d)$
Figura 2. Resultados da segmentação utilizando SLIC com diferentes quantidades de superpixels em uma imagem: (a) imagem original , (b) SLIC para 40 Segmentos, (c) SLIC para 100 Segmentos e (d) SLIC para 200 Segmentos.

\section{Proposta}

Neste trabalho propomos a identificação de eritrócitos utilizando a segmentação pela SLIC (Seção 2), e agrupamento por componentes conectados após processo de classificação supervisionada.

Nossa proposta é dividida em duas etapas: (i) uma imagem de entrada é segmentada pela SLIC, gerando um conjunto de superpixels. Em seguida o usuário informa quais superpixels são eritrócitos e quais não são eritrócitos. Esse processo de escolha será necessário para gerar a base de treinamento, que dará suporte a etapa de agrupamento e correção de regiões segmentadas de maneira incorreta. A Figura 3 apresenta o modelo de execução da primeira etapa. 

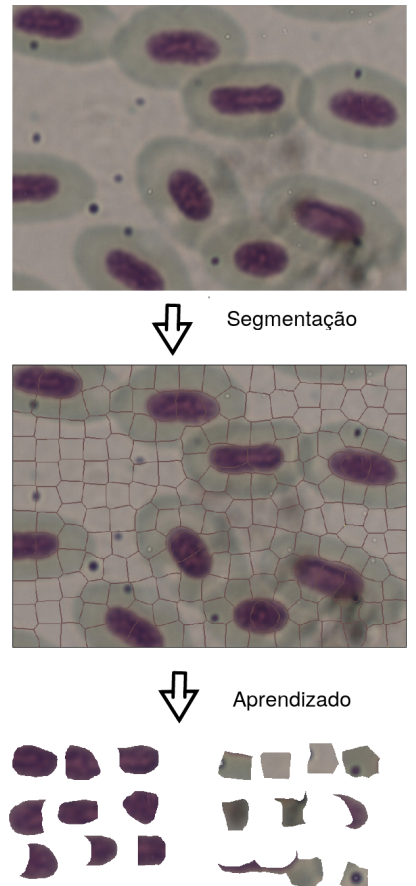

Eritrócitos

Não eritrócitos

Figura 3. Identificação manual de superpixels-eritrócitos para a geração de base de aprendizado.

Na segunda etapa, (ii) dada a imagem segmentada pela SLIC, executamos a classificação de cada superpixel, com base no conjunto de treinamento obtido pela primeira etapa. Conforme é classificado cada superpixel, a base de dados de treinamento armazena as informações para cada classe (superpixels eritrócito ou não-eritrócitos), e se algum superpixel for identificado incorretamente pela classificação, o usuário pode informar o erro, e adicionar a correção na base de aprendizado (mesmo procedimento da primeira etapa). Os passos para a identificação automática dos eritrócitos são apresentadas na Figura 4

Após esse processo, aplicamos então a etapa de agrupamento dos superpixels classificados. Nossa proposta, usa a técnica de agrupamento por componentes conectados [12], ou seja, geramos regiões de interesse usando abordagem baseada em grafos. Inicialmente, definimos superpixels como os componentes do grafo $\mathrm{G}=\{\mathrm{V}, \mathrm{A}\}$, onde $\mathrm{V}=\left\{v_{1} ; v_{2} ; \ldots\right.$; $\left.v_{n}\right\}$ conjunto de vértices, $\mathrm{A}=\left\{a_{1} ; a_{2} ; . . ; a_{m}\right\}$ o conjunto de arestas, sendo $\mathrm{p}\left(v_{i} ; v_{j}\right)$ o peso da aresta que conecta os vértices $v_{i}$ e $v_{j}$ com vizinhança de 4 pixels, e $n$ e $m$, o número de vértices e arestas, respectivamente.

Em seguida, nós agrupamos os superpixels que satisfazem um determinado limiar, obtendo um conjunto de regiões $\mathrm{R}=$ $\left\{r_{1} ; r_{2} ; \ldots ; r_{z}\right\}$, onde $z$ é o número total de regiões agrupadas. Se a condição do limiar for satisfeita as componentes $C_{i}$ e $C_{j}$ serão unidas em uma nova componente, se não, o algoritmo continua a execução verificando outras componentes. Assim, o processo é repetido até que o algoritmo não encontre mais nenhum par de componentes distintas que possuam arestas que

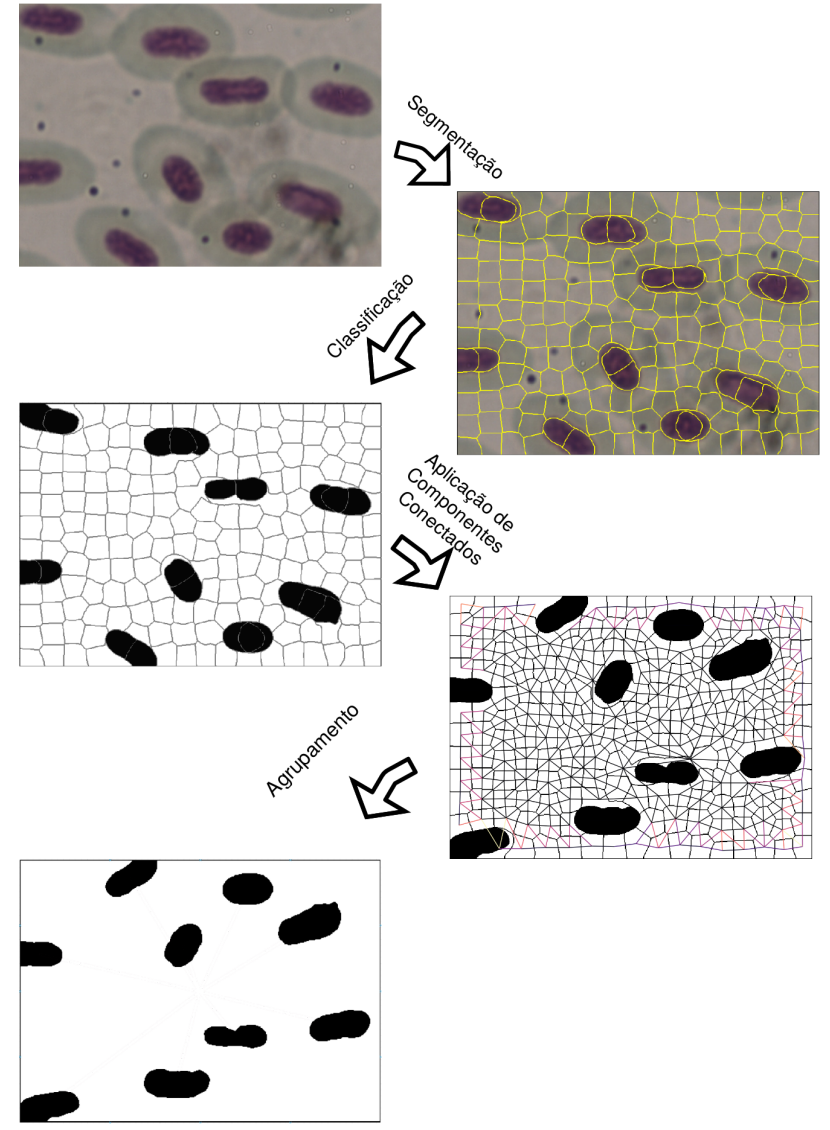

Figura 4. Identificação automática de eritrócitos.

satisfaçam essas condições, retornando as componentes como regiões propostas. Como cada superpixel poderá pertencer a apenas duas classes, ou seja, eritrócito ou não- eritrócitos, nossa condição de limiar é simplificado na condição de dois valores $\left(\mathrm{p}\left(v_{i} ; v_{j}\right)=0\right.$, representando dois superpixels eritrócito conectados e $\mathrm{p}\left(v_{i} ; v_{j}\right)=1$, representando dois superpixels de classes distintas conectadas). Com isso, cada região desse conjunto é definida por $r_{i}=\left\{s_{1} ; s_{2} ; \ldots ; s_{N r_{i}}\right\}$, onde $N r_{i}$ é o número total de superpixels da região $r_{i}$. Além disso, um superpixel $s_{i}$ só pertencerá a uma região $r_{i}$, se para um dado $j$, temos que $s_{j}$ percente $r_{i}$ e $s_{i}$ é vizinho de $s_{j}$.

Essa técnica se torna muito eficaz, já que precisamos aplicar uma regra de relação de adjacência, facilitando também o agrupamento dessas regiões.

\section{EXPERIMENTOS E RESULTADOS}

Para a realização dos testes para avaliação da proposta apresentada na Seção III, foi criado um banco de imagens onde foram selecionadas 326 imagens de 4 peixes. Para se fazer a coleta das imagens realizamos uma visita ao Laboratório de Ecotoxicologia e Genotoxicidade (LECOGEN) juntamente com os especialistas na área. No processo de aquisição de imagens, os especialistas injetaram um composto nos peixes conhecido como Ciclofosfamida $(40 \mathrm{mg} / \mathrm{Kg})$. Esse é um agente mutagênico do grupo das oxazoforinas que causa alterações 
em células sanguíneas. Esse composto é mais específico para material genético evitando a emissão de laudos falso-positivos o que torna as análises mais confiáveis.

As imagens foram coletadas com uma Câmera do Microscópio que fornece imagens com uma dimensão 2048 x 1536 pixels no formato JPEG, e organizadas em 4 categorias sendo: 24 horas, 48 horas, 72 horas e 96 horas. Essa separação foi feita justamente para tentarmos avaliar se o tempo que os peixes permanecem expostos no aquário pode interferir na contagem dos eritrócitos e alterar os resultados. A Figura 5 , apresenta exemplos de imagens de cada categoria.

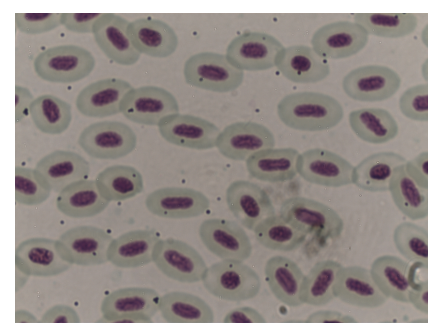

(a)

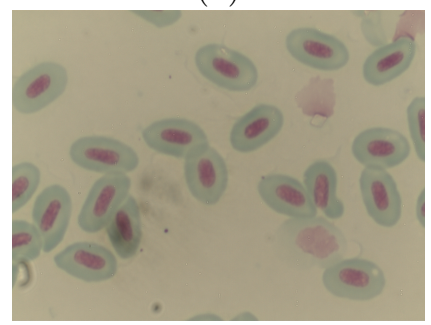

(c)

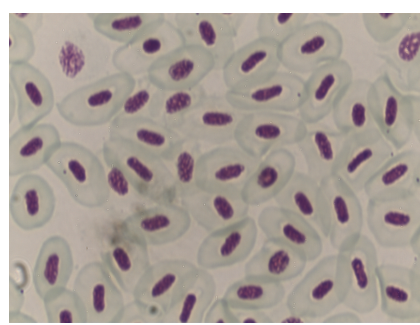

(b)

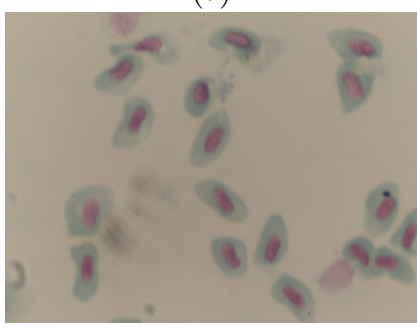

$(d)$
Figura 5. Imagens das categorias. (a) imagem da categoria 24 horas, (b) imagem da categoria 48 horas, (c) imagem da categoria 72 horas e (d) imagem da categoria 96 horas.

A proposta de detecção e identificação de eritrócitos foi desenvolvida utilizando a linguagem Python em conjunto com as bibliotecas Scikit-Image1, OpenCV e o WEKA. Para a avaliação da nossa proposta, iremos comparar os resultados com duas outras técnicas tradicionais na área: Template Matching: usada para encontrar instância de padrões de um kernel em imagens [13]; e SEG-IHC: realiza segmentação de objetos de interesse, usando mecanismos para identificação dos núcleos agrupados por DAB [14].

Inicialmente realizamos um processo de contagem manual, considerada em nossos experimentos como contagem do especialista para que pudéssemos comparar com os resultados da nossa proposta e das técnicas Template Matching e SEG-IHC. Nesta contagem manual foi utilizado um plugin do ImageJ, o Cell Counter $\eta^{2}$ que permite fazer marcações na imagem e contabilizar os eritrócitos. Após todo esse processo obtivemos nosso banco de dados organizado e com a contagem manual (especialista).

\footnotetext{
${ }^{1}$ É uma coleção de algoritmos para processamento de imagens, livre de restrições e código aberto - https://scikit-image.org/.

${ }^{2}$ https://imagej.nih.gov/ij/plugins/cell-counter.html
}

Para o processo de treinamento supervisionado da nossa proposta, foi utilizado 6 métodos de extração de atributos: histograma de gradiente orientado [15], matriz de coocorrencia [16], local binary patterns [17], 7 momentos de HU [18], estatísticas de cores no modelo RGB, e filtros de Gabor [19].

Com isso, para comparamos nossa proposta com as demais técnicas Template Matching e $S E G-I H C$ utilizamos 3 métricas:

- Recall: Consiste na divisão entre a quantidade de eritrócitos contados corretamente pela técnica $(h r)$ e a quantidade de eritrócitos contados pelo especialista (ec). Avaliado em uma escala de 0 a 1 , quanto mais próximo do valor 1 , indica que a técnica está detectando corretamente o maior número de eritrócitos existentes (Equação 3).

$$
\text { Recall }=\frac{h r}{e c}
$$

- Precision: Consiste na divisão entre a quantidade de eritrócitos contados corretamente pela técnica $(h r)$ e a quantidade de eritrócitos contados pela técnica $(h c)$. Avaliado em uma escala de 0 a 1 , quanto mais próximo do valor 1 , a técnica é mais precisa na realização da contagem (Equação 4).

$$
\text { Precision }=\frac{h r}{h c}
$$

- F-Score (F): Uma equação que balanceia os valores de Precision e Recall, obtendo um valor mais significativo para a análise. Um valor de Recall alto não significa que a técnica seja eficiente, pois a técnica pode detectar todos os eritrócitos, mas detectando vários pontos que não correspondem a um eritrócito, o que ocasionará em uma Precision baixa. Portanto, equilibrar estes valores é importante para a análise (Equação 5).

$$
F=2 * \frac{\text { Precision } * \text { Recall }}{\text { Precision }+ \text { Recall }}
$$

\section{AnÁlise dos Resultados}

As Tabelas I- III ilustram os resultados da proposta (nomeada de Prop $_{s l i c}$ ) em comparação com as técnicas Template Matching e SEG-IHC, respectivamente. Os resultados são apresentados já separados por categorias, e apresentados no seguinte formato $x \pm y$ onde $x$ é o valor da métrica e $y$ o valor de seu desvio padrão.

Tabela I

PROP $_{\text {slic }}$

\begin{tabular}{|c||c||c||c|}
\hline Categoria & Precision & Recall & F-Score \\
\hline 24h & $\mathbf{0 , 9 8 9} \pm \mathbf{0 , 3 1 7}$ & $0,980 \pm 0,071$ & $\mathbf{0 , 9 8 3} \pm \mathbf{0 , 0 4 4}$ \\
\hline $48 \mathrm{~h}$ & $\mathbf{0 , 9 5 4} \pm \mathbf{0 , 0 8 0}$ & $\mathbf{0 , 9 9 6} \pm \mathbf{0 , 0 1 6}$ & $\mathbf{0 , 9 7 4} \pm \mathbf{0 , 0 4 6}$ \\
\hline $72 \mathrm{~h}$ & $\mathbf{0 , 9 9 3} \pm \mathbf{0 , 0 2 1}$ & $0,958 \pm 0,075$ & $\mathbf{0 , 9 7 5} \pm \mathbf{0 , 0 4 7}$ \\
\hline 96h & $\mathbf{0 , 9 9 0} \pm \mathbf{0 , 0 2 5}$ & $0,926 \pm 0,129$ & $\mathbf{0 , 9 5 5} \pm \mathbf{0 , 0 8 5}$ \\
\hline
\end{tabular}


Tabela II

TEMPLATE MATCHING

\begin{tabular}{|c||c||c||c|}
\hline Categoria & Precision & Recall & F-Score \\
\hline $24 \mathrm{~h}$ & $0,844 \pm 0,078$ & $0,833 \pm 0,065$ & $0,839 \pm 0,071$ \\
\hline $48 \mathrm{~h}$ & $0,804 \pm 0,011$ & $0,833 \pm 0,115$ & $0,818 \pm 0,115$ \\
\hline $72 \mathrm{~h}$ & $0,847 \pm 0,116$ & $0,810 \pm 0,099$ & $0,828 \pm 0.106$ \\
\hline $96 \mathrm{~h}$ & $0,840 \pm 0,095$ & $0,833 \pm 0,095$ & $0,837 \pm 0,095$ \\
\hline
\end{tabular}

Tabela III

SEG-IHC

\begin{tabular}{|c||c||c||c|}
\hline Categoria & Precision & Recall & F-Score \\
\hline $24 \mathrm{~h}$ & $0,882 \pm 0,140$ & $\mathbf{0 , 9 8 5} \pm \mathbf{0 , 1 0 3}$ & $0,931 \pm 0,119$ \\
\hline $48 \mathrm{~h}$ & $0,863 \pm 0,142$ & $0,975 \pm 0,054$ & $0,916 \pm 0,078$ \\
\hline $72 \mathrm{~h}$ & $0,859 \pm 0,139$ & $\mathbf{1 , 0 0 0} \pm \mathbf{0 , 0 2 9}$ & $0,924 \pm 0,048$ \\
\hline $96 \mathrm{~h}$ & $0,808 \pm 0,112$ & $\mathbf{1 , 0 0 0} \pm \mathbf{0 , 0 4 1}$ & $0,894 \pm 0,060$ \\
\hline
\end{tabular}

Podemos observar que a proposta Prop $_{\text {slic }}$ apresentou os melhores resultados. Os valores de maior destaque para Recall foi para a categoria 48h, para Precision categoria $96 \mathrm{~h}$, e $F$ Score 24h. Para o Template Matching a melhor categoria de classificação em relação a Precision foi categoria $72 \mathrm{~h}$ e para Recall e $F$-Score a categoria de $24 \mathrm{~h}$ - Tabela II Analisando a métrica Recall, para 75\% das categorias SEG-IHC foi melhor dentre todas as técnicas. Isso se deve especialmente a escolha do limiar (SEG-IHC), pois apesar dos ruídos apresentados nas imagens (fato que deixou precision baixa), mantém sempre uma identificação satisfatória dos eritrócitos. Porém, caso haja uma alteração no padrão de detalhes dos eritrócitos, sua eficiência diminui, como apresentado na Tabela III] (categorias $24 h$ e $48 h$ ).

Realizando uma análise mais aprofundada, é possível comparar estatisticamente os pares dos resultados apresentadas de Prop slic com Template Matching, Prop slic com SEG$I H C$ e Template Matching com SEG-IHC, usando Wilcoxon signed rank test [22]. O teste de Wilcoxon é uma análise de grande importância pela capacidade de detectar diferenças mais sensíveis, uma vez que não assume distribuição normal. Neste caso, chegamos para o par das ferramentas de detecção Prop $_{\text {slic }}$ com Template Matching no resultado de análise com $p=0.002516$, sendo $(p<0.05)$ e Prop slic $_{\text {com }}$ SEG-IHC com $p=0.03418$. No caso de Template Matching com SEG-IHC, foi obtido um valor de $p=0.001465$, isso confirma que há diferença estatística significativa entre as técnicas de detecção avaliadas.

\section{CONCLUSÃO}

Neste trabalho foi apresentado uma proposta de identificação automática de eritrócitos, usando superpixels por SLIC e agrupamento por componentes conectados através do resultado de classificação supervisionada e comparamos os resultados com técnicas tradicionais como: Template Matching e SEG-IHC. Através da análise dos resultados dos experimentos e das imagens resultantes após o processamento das técnicas, verificou-se que a proposta foi eficiente em todas as categorias $(24 \mathrm{~h}, 48 \mathrm{~h}, 72 \mathrm{~h}$ e $96 \mathrm{~h})$ usando as métricas Precision e F-Score, com destaque para a técnica SEG-IHC apresentando os melhores resultados na métrica Recall para as categorias (24h, 72h e 96h). Trabalhos futuros visam o estudo de técnicas que resolvam os problemas de aglomeração de eritrócitos e ruídos nas imagens, além também de criar bases de treinamento para diferentes categorias. Durante a execução dos experimentos e contato com os especialistas foi identificado também um grande interesse na classificação automática dos eritrócitos, e sua contagem de maneira categorizada identificando possíveis mutações.

\section{REFERÊNCIAS}

[1] G. H. Merten and J. P. Minella, "Qualidade da água em bacias hidrográficas rurais: um desafio atual para a sobrevivência futura," Agroecologia e desenvolvimento rural sustentável, vol. 3, no. 4, pp. 3338, 2002.

[2] R. T. Silva and M. F. d. A. Porto, "Gestão urbana e gestão das águas: caminhos da integração," Estudos avançados, vol. 17, no. 47, pp. 129$145,2003$.

[3] J. M. Bewes, N. Suchowerska, and D. R. McKenzie, "Automated cell colony counting and analysis using the circular hough image transform algorithm (chita)," Physics in Medicine Biology, vol. 53, no. 21, p. 5991, 2008. [Online]. Available: http://stacks.iop.org/0031-9155/53/i= $21 / \mathrm{a}=007$

[4] J. Byun, M. R. Verardo, B. Sumengen, G. P. Lewis, B. Manjunath, and S. K. Fisher, "Automated tool for the detection of cell nuclei in digital microscopic images: application to retinal images," Mol Vis, vol. 12, no. 105-07, pp. 949-60, 2006.

[5] K. Souza, A. Pinheiro, W. Amorim, and V. Odakura, "Contagem automática de unidades formadoras de colônias de bactérias em placas de petri com o uso do algoritmo template matching," 2012.

[6] R. Achanta, A. Shaji, K. Smith, A. Lucchi, P. Fua, and S. Süsstrunk, "Slic superpixels compared to state-of-the-art superpixel methods," IEEE transactions on pattern analysis and machine intelligence, vol. 34, no. 11 , pp. 2274-2282, 2012.

[7] A. Trémeau and P. Colantoni, "Regions adjacency graph applied to color image segmentation," IEEE Transactions on image processing, vol. 9, no. 4, pp. 735-744, 2000.

[8] P. F. Felzenszwalb and D. P. Huttenlocher, "Efficient graph-based image segmentation," International journal of computer vision, vol. 59, no. 2, pp. 167-181, 2004.

[9] R.-F. Chang, C.-J. Chen, and C.-H. Liao, "Region-based image retrieval using edgeflow segmentation and region adjacency graph," in Multimedia and Expo, 2004. ICME'04. 2004 IEEE International Conference on, vol. 3. IEEE, 2004, pp. 1883-1886.

[10] P. Janaina, Detecção da citotoxicidade, genotoxicidade e mutagenicidade, do inseticida fipronil no organismo teste Allium cepa. Dissertação, Instituto de Biociências, Universidade Estadual Paulista, 2008.

[11] K. Saarinen, "Color image segmentation by a watershed algorithm and region adjacency graph processing," in Image processing, 1994. Proceedings. ICIP-94., IEEE international conference, vol. 3. IEEE, 1994, pp. 1021-1025.

[12] F. Chung and L. Lu, "Connected components in random graphs with given expected degree sequences," Annals of combinatorics, vol. 6, no. 2, pp. 125-145, 2002.

[13] F. Jurie and M. Dhome, "A simple and efficient template matching algorithm," in Computer Vision, 2001. ICCV 2001. Proceedings. Eighth IEEE International Conference on, vol. 2. IEEE, 2001, pp. 544-549.

[14] J. Shu, H. Fu, G. Qiu, P. Kaye, and M. Ilyas, "Segmenting overlapping cell nuclei in digital histopathology images," in 2013 35th Annual International Conference of the IEEE Engineering in Medicine and Biology Society (EMBC). IEEE, 2013, pp. 5445-5448.

[15] N. Dalal and B. Triggs, "Histograms of oriented gradients for human detection," in Computer Vision and Pattern Recognition, 2005. CVPR 2005. IEEE Computer Society Conference on, vol. 1. IEEE, 2005, pp. 886-893.

[16] A. K. C. Wong and M. A. Vogel, "Textural features for image classification," IEEE Transactions on systems, man, and cybernetics, no. 6, pp. 610-621, 1973. 
[17] T. Ojala, M. Pietikainen, and T. Maenpaa, "Multiresolution gray-scale and rotation invariant texture classification with local binary patterns," IEEE Transactions on pattern analysis and machine intelligence, vol. 24, no. 7, pp. 971-987, 2002.

[18] K. P. Souza and H. Pistori, "Implementaç ao de um extrator de caracteristicas baseado em momentos da imagem."

[19] N. P. Angelo, "Aplicação de filtros de gabor no processo de classificação de imagens digitais com base em atributos de textura," 2000.

[20] P. L. R. Dourado, Biomonitoramento Da Qualidade De Água Superficial Em Áreas Agroindustriais No Mato Grosso Do Sul Dourados/MS, Brasil: UFGD Faculdade De Ciências Exatas E Tecnologias, 2014.

[21] Ramsdorf WA, Ferraro MVM, Oliveira-Ribeiro CA, Cestari MM Genotoxic evaluation of different doses of inorganic lead (PbII) in Hoplias malabaricus. Environ Monit Assess, 2009.

[22] J. Demšar, "Statistical comparisons of classifiers over multiple data sets," J. Mach. Learn. Res., vol. 7, pp. 1-30, Dec. 2006. [Online]. Available: http://dl.acm.org/citation.cfm?id=1248547.1248548 